\title{
Manipulación nutricional en el camarón Macrobrachium borellii del río Paraná (Argentina) como recurso para la alimentación humana
}

\author{
Maria Eugenia D'Alessandro ${ }^{1}$ y Pablo Collins, ${ }^{2, *}$ \\ ${ }^{1}$ Facultad de Bioquímica y Ciencias Biológicas, Universidad Nacional del Litoral (CONICET-UNL) Argentina. \\ 2 Instituto Nacional de Limnología, Universidad Nacional del Litoral (CONICET-UNL) Argentina. \\ * Corresponding author: pagcollins@gmail.com
}

Received: 05/11/18 Accepted: 30/09/19

\section{RESUMEN}

\section{Manipulación nutricional en el camarón Macrobrachium borellii del río Paraná (Argentina) como recurso para la alimentación humana}

Los ambientes dulciacuícolas aportan una gran diversidad de hábitats y recursos, y proveen servicios a la sociedad. Entre estos servicios, el valor nutricional es importante. Los crustáceos, particularmente los camarones, proveen un potencial alimento con características nutricionales reconocidas por su alto contenido proteico. El camarón dulciacuícola Macrobrachium borellii en el río Paraná (América del Sur) llega a tener altas densidades. La especie es omnívora y oportunista, obteniendo los nutrientes necesarios del ambiente que se reflejan en el perfil de aminoácidos (AA) que caracteriza a la especie. En el contexto del servicio que ofrece esta especie, el objetivo de este trabajo fue evaluar el perfil de AA del músculo del camarón $M$. borellii (porción comestible) obtenido del ambiente y cultivados con diferentes dietas isoproteicas, en función de promover su cultivo para consumo humano y preservar el recurso natural. Ejemplares de $M$. borellii fueron capturados del valle de inundación del río Paraná y cultivados en tanques durante tres meses. En los cultivos fueron alimentados con tres dietas pelletizada que contenían harinas de origen animal y vegetal en diferentes proporciones. El contenido de proteínas, lípidos, hidratos de carbono y de agua fueron evaluados. A su vez, el perfil de aminoácidos de las dietas administradas y del músculo de los camarones cultivados y provenientes del ambiente fueron analizados por HPLC y comparados con el requerimiento de AA indispensables para adultos. En el músculo del camarón $M$. borellii se registraron 16 AA, ocho de ellos fueron AA indispensables y ocho AA no indispensables. Los AA Asp y Glu fueron los más abundantes en los animales cultivados. Los valores de concentración de los AA azufrados (Cys+Met), ramificados (Val+Leu+Ile), aromáticos (Tyr+Phe) y aminados (Lis+Arg), muestran valores de relevancia en nutrición. Al comparar el patrón de AA indispensables para el hombre en su condición de niño-adolescente y adulto, calculado con un valor de requerimiento proteico promedio de 0.80 y $0.66 \mathrm{~g} \mathrm{~kg}^{-1} \mathrm{~d}^{-1}$ respectivamente, con aquellos provistos por el músculo de los camarones provenientes del río Paraná y de aquellos cultivados con las tres dietas no se encontraron AA limitantes. La porción comestible de los camarones cultivados cubre los requerimientos de AA indispensables para los humanos y constituye una fuente potencial de nutrientes.

Palabras clave: perfil de aminoácidos, acuicultura, nutrición humana, servicio ecosistémico

\section{ABSTRACT}

\section{Nutritional manipulation in the prawn Macrobrachium borellii of Paraná River as a resource for the human feeding}

The freshwater environments have a great diversity of habitats and resources providing services to society. Among the services the nutritional value of resources is important. Crustaceans, particularly prawns, supply human beings with food resources with high protein content. The prawn Macrobrachium borellii inhabits the Paraná River (South America) in high densities. The species is omnivorous and opportunistic, obtaining their nutrients from the environment, which is reflected in a characteristic amino acid profile (AA). Keeping in mind the valorization of ecosystem service, our objective was to evaluate the AA profile of the prawn M. borellii muscle (edible portion) obtained from the environment and from cultivation with different iso-protein diets promote its cultivation for human consumption and preserve the species. Specimens of $\mathrm{M}$. borellii were captured from the floodplain of the Paraná River and cultivated in tanks for three months. The cultivated prawns were fed with three pelletized 
diets containing flours of animal and plant origin in different proportions. The contents of proteins, lipids, carbohydrates, and humidity were evaluated. The amino acid profile of the diets and the muscle of the cultivated prawn and from the environment were analyzed by HPLC and compared with the requirement of AA essential for child-adolescents and adults. In the muscle, 16 AA were recorded, eight were essential and eight not essential. Asp and Glu were the most abundant AA in the cultivated specimens. Sulfurized AA (Cys + Met), branched (Val + Leu + Ile), aromatic (Tyr + Phe) and aminated (Lis + Arg), were high. When comparing essential AA patterns for child-adolescent humans and adults, calculated with an average protein requirement value of 0.80 and $0.66 \mathrm{~g} \mathrm{~kg}^{-1} \mathrm{~d}^{-1}$ respectively for specimens from the Paraná River, and from those cultivated with the three diets, we found no limiting AA. The edible portion of the cultivated specimens covers the requirements of AA essential for humans, therefore, the cultivation of this species with artificial diets is a potential nutritional resource for humans.

Key words: aminoacids profile, aquaculture, human nutrition, ecosystem services

\section{INTRODUCCIÓN}

Los grandes ríos de llanura con valle de inundación presentan una gran diversidad de ambientes y especies. Estas viven en sistemas que están gobernados por variables estacionales como son los ciclos climáticos e hidrosedimentarios (Junk et al., 1989; Iriondo et al., 2007). Con esta variación sistemática, los organismos obtienen energía y materia dentro de tramas tróficas que se muestran dinámicas (Winemiller, 2004), en donde las macromoléculas que caracterizan a cada especie son transferidas de un nivel trófico al siguiente, y a éste le sirve de base para establecer el perfil de macromoléculas propio que le permitirá realizar las funciones que le garantice su existencia en ese ambiente (Frost et al., 2005; Saborowski, 2015).

La estacionalidad marca que los organismos ajustan sus perfiles de macromoléculas a los presentes en los recursos tróficos y así mismo a modificaciones ontogenéticas (Laughlin, 1982; Collins, 2019). Algunas especies son altamente selectivas en el tipo de alimento que consumen durante todo el año, mientras que otras tienen facilidades de aprovechar una gran variedad de recursos provistos por el ambiente; éstas son conocidas como omnívoras (Krivan \& Diehl, 2005).

Dadas estas características y la necesidad de obtener alimentos de calidad para las sociedades humanas, la manipulación trófica es una herramienta en la acuicultura que permite obtener productos con características específicamente diseñadas. Además, y como un recurso de valoración, la acuicultura usa la calidad nutricional de sus productos como propiedad para jerarquizar los cultivos, siendo un beneficio de esta activi- dad. A esto se le suman la bioseguridad del producto cultivado, predictibilidad de la producción, y aquellos aspectos relacionados a la conservación del recurso y manipulación de ellos (FAO, 1997).

La omnivoría es un tipo de alimentación difícil de emular en todas sus características o valoraciones debido a que los animales obtienen los nutrientes de una gran diversidad de recursos de sus ambientes naturales. En los sistemas simplificados, como son los cultivos, la diversidad de recursos tróficos está limitada por el tipo de alimento que se formule y los recursos que se generen en los sistemas de cultivo (Collins, 1999). La seguridad de proveer en un cultivo la mayoría de los nutrientes necesarios es una búsqueda constante en las investigaciones, más aún, obtener especies cultivadas que contengan las características del alimento deseado, mejorando o por lo menos alcanzando la calidad del animal obtenido del ambiente, es un desafío.

Un componente biológico de los ecosistemas acuáticos son los crustáceos, que tienen una característica invaluable reconocida por su alta cantidad de proteínas y bajo contenido lipídico (García-Guerrero et al., 2003; Ramirez et al., 2010; Rangappa et al., 2012; Islam et al., 2017). Entre los crustáceos, los camarones y langostinos son un recurso para los humanos por las macromoléculas que se encuentran en sus partes comestibles, y cuya calidad y cantidad pueden ser manipuladas. El patrón de aminoácidos (AA) y de ácidos grasos debe considerarse como un recurso biológico que podrían aportar factores nutricionales que permita un mejoramiento en la salud humana (Elmadfa \& Meyer, 2017).

En este contexto es necesario reconocer que 
los animales producen proteína a partir de la conversión de diferentes tipos de moléculas que toman del ambiente. El crustáceo Macrobrachium borellii, un camarón de agua dulce que habita en el río Paraná, transforma en proteínas las macromoléculas provenientes de sus recursos tróficos, como restos vegetales, hongos, microorganismos, larvas de insecto, oligoquetos, peces y otros componentes de la biota del río y su valle de inundación (Collins \& Paggi, 1998). Los omnívoros son especies deseadas para su cultivo por la facilidad en aceptar el alimento. Sin embargo, formular un alimento artificial que provea todos los nutrientes es complejo, y más aún que el producto final presente un patrón similar en sus moléculas que el de los animales provenientes del ambiente (Tacon, 2016).

El objetivo de este trabajo fue evaluar la capacidad del camarón Macrobrachium borellii de incorporar aminoácidos desde los alimentos suministrados artificialmente y que la porción comestible presente similar perfil de AA al de los animales que viven en el ambiente natural, pudiendo reconocer la capacidad de enriquecer o empobrecer alguno de ellos con el fin de proveer cantidad y calidad de proteínas de valor para el consumo humano.

\section{MATERIAL Y MÉTODOS}

\section{Captura de animales}

Los camarones fueron capturados en el valle de inundación del río Paraná. Ejemplares juveniles de similares tallas fueron seleccionados para la inclusión en las experiencias. La captura se realizó al inicio de la primavera (octubre de 2017) con red de mano de $2 \mathrm{~mm}$ de abertura de poro, y los camarones se trasladaron inmediatamente a los laboratorios del Instituto Nacional de Limnología, Santa Fe Argentina.

\section{Sistema de cultivo y manejo}

Los ejemplares seleccionados en intermuda de largo de cefalotórax (LC) entre 8.7 y $11.3 \mathrm{~mm}$ fueron colocados en nueve tanques circulares de fibra de vidrio de 200 1, a una densidad de 120 $\mathrm{ind} / \mathrm{m}^{2}$ o $1 \mathrm{ind} / 51$. Los tanques tuvieron circula-
Tabla 1. Composición proximal de las dietas utilizadas durante la experiencia de cultivo de Macrobrachium borellii durante tres meses (media \pm desvío estándar). Proximal composition of the diets used during the Macrobrachium borellii cultivation experience for three months (mean \pm standard deviation).

\begin{tabular}{lccc}
\hline Dieta & D1 & D2 & D3 \\
\hline Proteína & $20.04 \pm 4.21$ & $21.87 \pm 4.29$ & $21.37 \pm 2.90$ \\
Lípidos & $5.85 \pm 1.99$ & $6.53 \pm 1.10$ & $5.95 \pm 0.85$ \\
Hidratos de carbono & $0.60 \pm 0.16$ & $0.60 \pm 0.22$ & $0.45 \pm 0.85$ \\
Humedad & $79.74 \pm 0.98$ & $80.63 \pm 0.34$ & $80.45 \pm 1.39$ \\
\hline
\end{tabular}

ción de agua, aireación constante, fotoperiodo $12: 12 \mathrm{~h}$, y temperatura de $22 \pm 2{ }^{\circ} \mathrm{C}$. A los camarones se les ofreció diariamente alimento peletizado en una ración del $10 \%$ de la biomasa de cada tanque.

Durante tres meses se ofreció tres dietas isoproteicas (Tabla 1), variando el porcentaje de harinas de origen vegetal y animal, y de celulosa como elemento inerte (harinas de pescado $\mathrm{y}$ camarón, harinas de soja, trigo, y maíz, vitaminas y minerales, y aceite de pescado). Diariamente, a través de aspiración, se limpió el fondo de los tanques retirando el alimento no consumido, mudas, y animales muertos. Luego de ello, se procedió a la alimentación.

\section{Datos ambientales y morfométricos}

En cada tanque se registró a diario la temperatura, oxígeno disuelto y $\mathrm{pH}$, y cada tres días se midió nitratos y nitritos mediante un medidor multiparamétrico HACH HQ40d. Además, diariamente se observó las mudas e individuos muertos. Los ejemplares fueron medidos (LC) con un calibre digital $( \pm 0.01 \mathrm{~mm})$ desde la punta del rostro hasta el extremo posterior del cefalotórax y pesados en una balanza digital $( \pm 0.01 \mathrm{~g})$.

El contenido de proteínas, patrón o perfil de aminoácidos, y humedad relativa se determinó en músculo del abdomen de los ejemplares extraídos del ambiente, de aquellos cultivados con las tres dietas al finalizar la experiencia y de los alimentos suministrados. Los músculos analizados fueron diseccionados del abdomen en frío $\left(2{ }^{\circ} \mathrm{C}\right)$ y mantenidos a $-20{ }^{\circ} \mathrm{C}$ hasta realizar las determinaciones. 
Las proteínas se cuantificaron por microKjeldahl, los lípidos por Soxhlet y la humedad por secado en estufa a $105^{\circ} \mathrm{C}$ por 24 horas. El perfil de aminoácidos del músculo y alimentos se realizó por hidrolisis ácida (Alaiz et al., 1992), y analizadas en HPLC después de que las muestras fueran derivatizadas con dietiletoximetilen malonato.

\section{Patrón de requerimientos de aminoácidos en los humanos}

Para reconocer la calidad de las proteínas presentes en el músculo de los camarones provenientes del ambiente y de los cultivos y su valoración para la alimentación humana se comparó el patrón de aminoácidos obtenidos de los camarones con el patrón de requerimientos mínimos de los aminoácidos indispensables para los seres humanos (fuente: WHO/FAO/UNU, 2007) para los grupos etarios niño-adolescente y adulto. Esto se calculó reconociendo el valor de requerimiento proteico promedio de $0.80 \mathrm{~g} \mathrm{~kg}^{-1} \mathrm{~d}^{-1}$ y $0.66 \mathrm{~g} \mathrm{~kg}^{-1}$ $\mathrm{d}^{-1}$ respectivamente (Tabla 2 ).

\section{Análisis de datos}

Los valores de calidad del sistema de cultivo (temperatura, $\mathrm{pH}$, oxígeno disuelto, nitritos $\mathrm{y}$ nitratos) fueron evaluados mediante Kruskal Wallis y post - test de Mann Whitney (Zar, 1996). El LC de los individuos cultivados de cada tanque

Tabla 2. Patrón de aminoácidos indispensables para adolescentes y adultos humanos tomando como requerimiento proteico el valor de 0.80 y $0.66 \mathrm{~g} \mathrm{~kg}^{-1} \mathrm{~d}^{-1}$ respectivamente (WHO / FAO / UNU, 2007). Essential amino acid pattern for adolescents and human adults, taking as a protein requirement the value of 0.80 and $0.66 \mathrm{~g} \mathrm{~kg}^{-1} \mathrm{~d}^{-1}$ respectively (WHO / FAO / UNU, 2007).

\begin{tabular}{lcc}
\hline & $\begin{array}{c}\text { Patrón niños escolares } \\
\text { /adolescentes }\end{array}$ & Patrón adultos \\
\hline Histidina & 16 & 15 \\
Treonina & 25 & 23 \\
Fenilalanina / Tirosina & 41 & 38 \\
Valina & 40 & 39 \\
Metionina / Cisteína & 23 & 22 \\
Isoleucina & 30 & 30 \\
Triptófano & 7 & 6 \\
Leucina & 61 & 59 \\
Lisina & 48 & 45 \\
\hline
\end{tabular}

y como éste varió en el tiempo fue comparado por ANOVA de una vía (Zar, 1996).

El contenido de cada aminoácido fue comparado entre los individuos obtenidos del ambiente y aquellos que estuvieron en los estanques luego de tres meses de experiencia alimentados con distintas dietas. El perfil de AA de estos individuos se los comparó con el del alimento suministrado. Además, el patrón de requerimiento de niños-adolescentes y adultos se comparó con los patrones obtenidos de los camarones capturados del ambiente y de aquellos alimentados con las diferentes dietas. Las diferencias entre los valores fueron comparadas mediante chi cuadrado estableciendo el límite inferior y superior que puede reconocerse (Zar, 1996).

\section{RESULTADOS}

\section{Sistema de cultivo}

Las condiciones del cultivo se mantuvieron estables durante toda la experiencia y en todos los tanques, oscilando la temperatura en $22 \pm 2{ }^{\circ} \mathrm{C}$, conductividad en $134 \pm 23 \mu \mathrm{S} / \mathrm{cm}$; oxígeno disuelto en $7.40 \pm 0.87 \mathrm{mg} / \mathrm{L}$, $\mathrm{pH}$ de $7.3 \pm 0.3$, nitrato $0.32 \pm 0.03 \mathrm{mg} / \mathrm{L}$, y nitrito menor a $0.05 \mathrm{mg} / \mathrm{L}$.

La talla y peso fueron similares en todos los tanques al finalizar la experiencia $(p>0.05)$ como también la supervivencia $(p>0.05)$, siendo esta última de $78 \%$ en los estanques de los camarones alimentados con la dieta D2, y $83.3 \%$ en aquellos que se les suministró las dietas D1 y D3.

\section{Perfil de AA del músculo del camarón Macro- brachium borellii obtenido del ambiente}

En el músculo del camarón $M$. borellii se registraron $16 \mathrm{AA}$, siendo $8 \mathrm{AA}$ indispensables y $8 \mathrm{AA}$ no indispensables. Este número se modifica a 9 y 7 respectivamente de acuerdo a si se considera Arg como indispensable. Considerando a Arg dentro de este grupo, el $54 \%$ correspondieron a estos AA y el $46 \%$ a los AA no-indispensables.

Los picos en el cromatograma del HPLC correspondientes a Asp y Glu son similares y no se pudieron separar por los que se los consideró como un solo registro. 


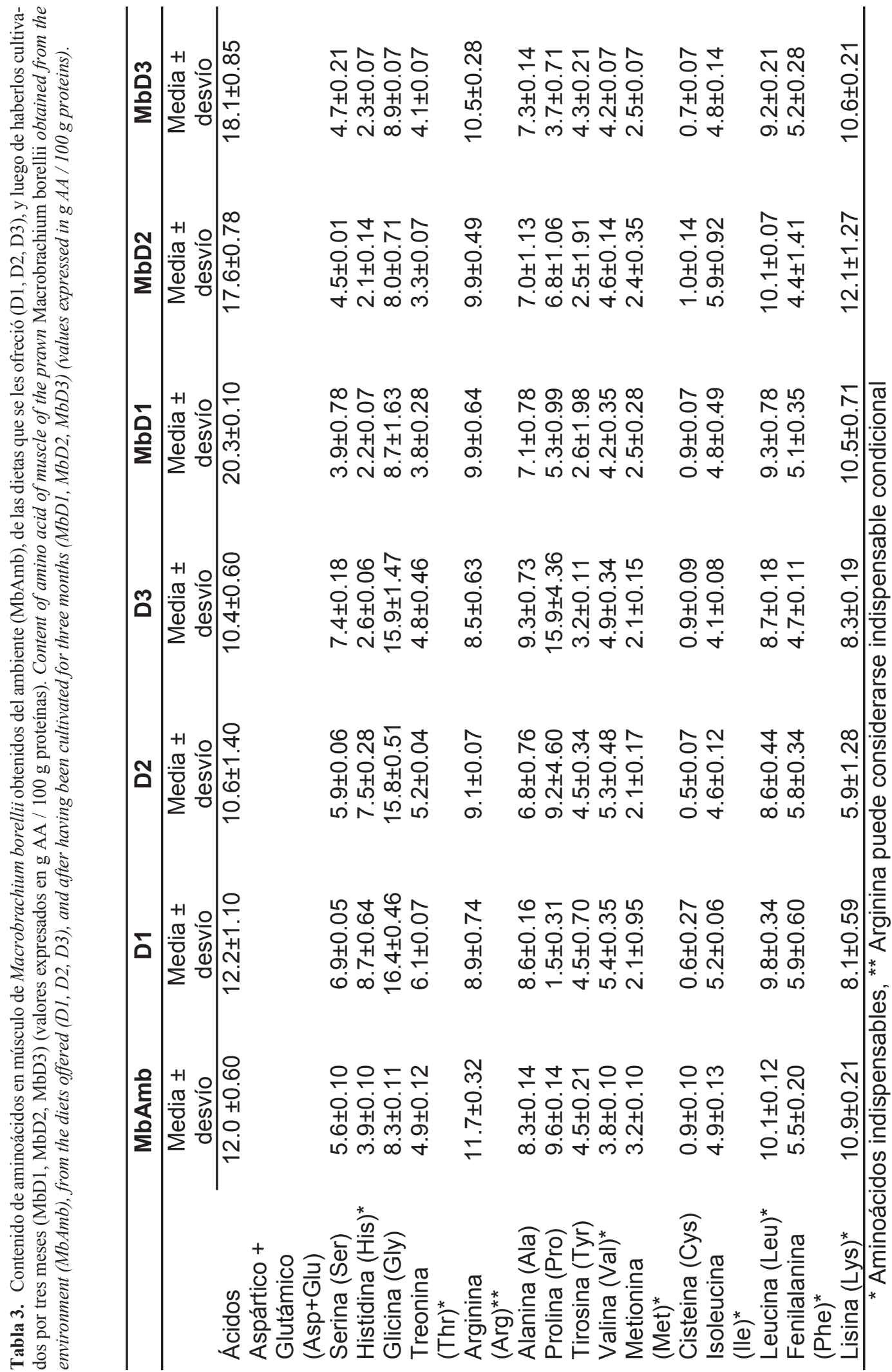


El orden decreciente de concentración de AA en animales en intermuda fue Asp $+\mathrm{Glu}>\mathrm{Arg}>$ Lys $>$ Leu $>$ Pro $>$ Gly $=$ Ala $>$ Ser $=$ Phe $>$ Thr $=$ Ile $>$ Tyr $>$ His $=$ Val $>$ Met $>$ Cys (Tabla 3$)$.

\section{Perfil de AA de las dietas y comparación con los AA del músculo de Macrobrachium borellii del ambiente}

Las dietas fueron isoproteicas (Tabla 1) pero difirieron en el perfil de AA (Tabla 3), si bien estaban presentes los $16 \mathrm{AA}$ que presentaron los individuos en el ambiente natural, los porcentajes de cada uno de ellos fue diferente.

Las tres dietas contenían más Ser, Gly, y Val, mientras que las dietas D1 y D2 tuvieron comparativamente más His, Thr, y Phe que los valores presentes en los músculos de los camarones provenientes del ambiente. Por otra parte, la dieta D3 tuvo concentraciones menores de esos AA (Fig. 1a). La dieta D1 contenía más Ile que el observado en los músculos de los camarones obtenidos del río Paraná. Mientras que los AA
Arg, Met, Cys y Lys presentaron valores de concentración menores en las tres dietas que los registrados en los músculos de los ejemplares provenientes del ambiente (Fig. 1a). Los valores de Ala, y Pro son significativamente diferentes en las tres dietas $(p<0.05)$.

\section{Aminoácidos en músculo de Macrobrachium borellii cultivados y comparación con las dietas suministradas}

En los camarones cultivados se observó valores mayores de los AA Asp+Glu, Arg, Met y Lys que los proporcionado en las tres dietas (Fig. 1b). El AA Pro solo estuvo presente con valores mayores en el músculo de los animales que se les ofreció la D1, y los animales alimentados con la D3 tuvieron más Tyr. Además, los camarones a los que se ofrecieron como alimentos las dietas D1 y D2 tuvieron más Cys, mientras que los animales en D2 y D3 presentaron más Ile y Leu (Fig. 1b). Por otra parte, en todos los cultivos (D1, D2 y D3) se observaron que los camarones presentaron en sus
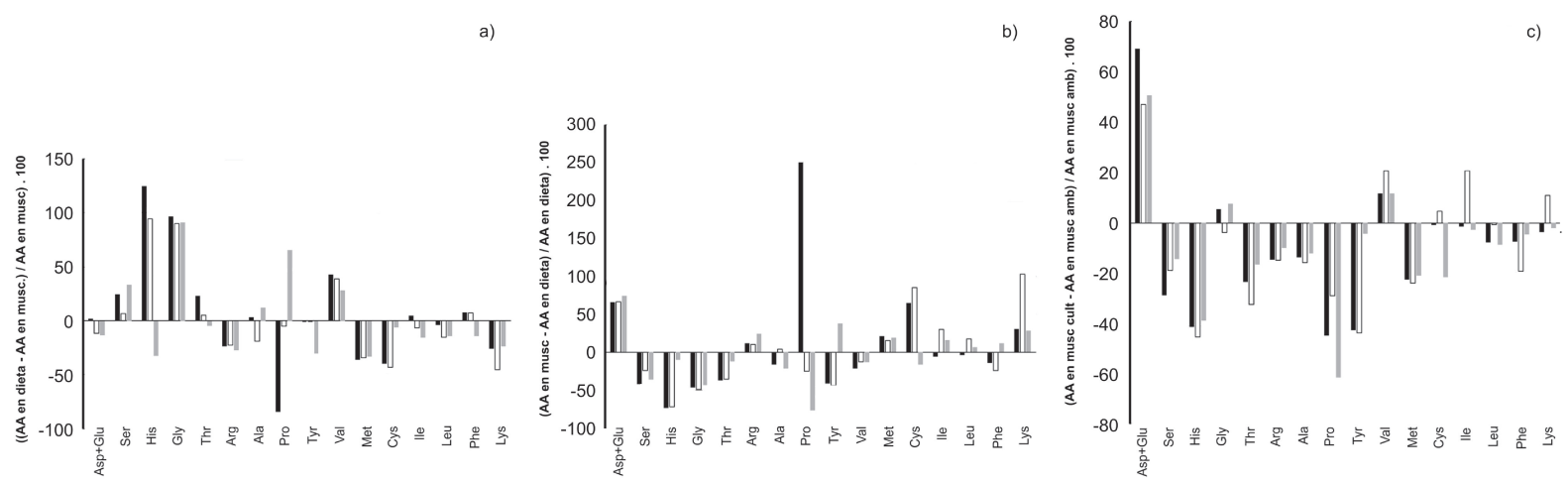

Figura 1. Relaciones entre: a) aminoácidos en las dietas y el músculo del camarón Macrobrachium borellii obtenidos del río Paraná (columna negra: D1-ambiente, columna blanca: D2-ambiente, columna gris: D3-ambiente); b) aminoácidos en las dietas y los camarones cultivados y alimentados con cada una de ellas (columna negra: músculo del camarón alimentados con D1-D1, columna blanca: músculo del camarón alimentados con D2-D2, columna gris: músculo del camarón alimentados con D3-D3); y c) aminoácidos presentes en el músculo del camarón alimentado con las dietas y músculo del camarón proveniente del río Paraná (columna negra: músculo del camarón alimentados con D1-músculo del camarón del ambiente, columna blanca: músculo del camarón alimentados con D2-músculo del camarón del ambiente, columna gris: músculo del camarón alimentados con D3-músculo del camarón del ambiente). Relationships between: a) amino acids in diets and in the muscle of the prawn Macrobrachium borellii obtained from the Paraná River (black column: D1-environment, white column: D2-environment, gray column: D3-environment); b) amino acids in the diets and what incorporated into the muscle of the prawn cultured and fed with each diet (black column: prawn muscle fed with D1-D1, white column: prawn muscle fed with D2-D2, gray column: prawn muscle fed with D3-D3); and c) amino acids present in the muscle of the prawn fed with each diets and muscle of the prawn from environment the Paraná River (black column: prawn muscle fed with D1-environment prawn muscle, white column: prawn muscle fed with D2-environment prawn muscle, gray column: prawn muscle fed with D3-environment prawn muscle). 
músculos menos concentraciones de los AA Ser, His, Gly, Thr y Val (Fig. 1b).

\section{Comparación del contenido de AA del cama- rón Macrobrachium borellii cultivados y obte- nidos del ambiente}

El contenido de Asp+Glu estaba en mayor concentración en los músculos de los animales alimentados con las tres dietas que lo que contenía los músculos de los animales provenientes del ambiente, lo mismo se observó con Val. Mientras que los animales alimentados con las dietas D1 y D3 tuvieron en sus músculos mayor concentración de Gly, y solo D2 provocó que los animales cultivados tuvieran valores mayores de Cys, Ile y Lys (Fig. 1c).

Por otra parte, Ser, Hys, Thr, Arg, Ala, Pro, Tyr, Mel, y Phe estuvieron en concentración mayor en los músculos de los ejemplares proveniente del ambiente que aquellos cultivados con las tres dietas (Fig. 1c).

\section{Comparación de los AA indispensables presentes en el camarón Macrobrachium bore- llii con los requerimientos del hombre}

Al comparar el perfil de AA indispensables con el patrón de requerimiento en el niño-adolescente y adulto se observa que el contenido en el músculo de los ejemplares provenientes del ambiente y aquellos cultivados superan los requerimientos mínimos que se indican para ellos (Fig. 2). Los camarones del ambiente tuvieron más Hys, Thr, y Met que los cultivados. Mientras que los animales alimentados con la dieta D2 aportaron más cantidad de Ile y Lys, y menos Phe. Sin embargo, Val y Leu fueron similares en los valores que ofrecen los camarones provenientes del ambiente y aquellos cultivados con las tres dietas (Fig. 2).

\section{DISCUSIÓN}

La dinámica del río, y en particular aquellos que presentan llanura con valle de inundación, provee a las comunidades biológicas condiciones que les permite desarrollarse con una determinada fenología (Frost et al., 2005; Collins, en prensa). La ocurrencia de un recurso trófico en un determina-

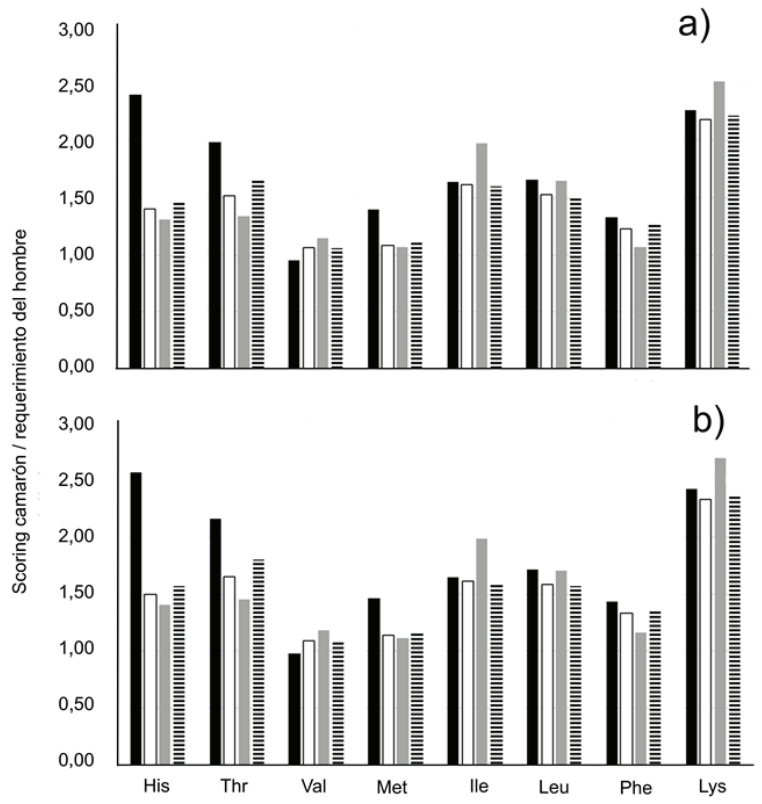

Figura 2. Relación entre el patrón de aminoácidos indispensables presentes en el músculo del camarón Macrobrachium borellii y el requerimiento para niño-adolescentes (a) y adultos (b). (columna negra: músculo del camarón del ambiente-hombre, columna blanca: músculo del camarón alimentados con D1-hombre, columna gris: músculo del camarón alimentados con D2-hombre, columna a rayas: músculo del camarón alimentados con D3-hombre. Relationship between the essential amino acid pattern present in the muscle of the prawn Macrobrachium borellii and the requirement for children-adolescents (a) and adults (b). (black column: muscle from environment prawn-man, white column: prawn muscle fed with D1-man, gray column: prawn muscle fed with D2-man, striped column: prawn muscle fed with D3-man).

do momento hace que las especies que lo consumen expresen en sus perfiles de macromoléculas las características intrínsecas de las especies utilizadas como alimento dentro de su trama trófica (Carvalho et al., 2016).

Por ello, los atributos nutricionales que provienen de la captación y transformación de los recursos tróficos obtenidos del ambiente caracterizan a las especies y son transformados para responder a las necesidades ontogenéticas y temporales dentro del ciclo natural de los sistemas acuáticos (Blanchette et al., 2014). Frente a esta situación, los organismos omnívoros expresan ciertas ventajas al poder utilizar una gran variedad de recursos y transformarlos para 
responder a sus necesidades. Esa multiplicidad llega a tal extremo de utilizar los elementos obtenidos del ambiente tanto como organismos vivos como también muertos (Collins et al., 2006).

En este trabajo se mostró un perfil de AA $($ Asp + Glu $>$ Arg $>$ Lys $>$ Leu $>$ Pro $>$ Gly $=$ Ala $>$ Ser $=$ Phe $>$ Thr $=$ Ile $>$ Tyr $>$ His $=$ Val $>$ Met $>$ Cys) que caracterizó al camarón $M$. borellii obtenido del ambiente, y por otro lado se reconoció que esta especie capta y selecciona algunos AA compensando la deficiencia presente en los alimentos al compararlos con el perfil de los animales provenientes del río. Los alimentos brindados en las experiencias presentaban diferencias en sus perfiles de AA, y con al menos tres meses de utilizar estos alimentos lograron modificar sus perfiles de AA en el músculo para responder a sus requerimientos. Estos son aspectos que permiten entender que ese perfil es dinámico y que caracteriza al momento y las fuentes de recurso trófico potencialmente utilizados como alimento por el camarón durante el ciclo estacional del sistema acuático.

En esta experiencia se observó que aquellos animales alimentados con dietas ofrecidas en la experiencia presentaron mayor proporción de AA azufrados que los provenientes del ambiente. Lo que indica que hubo una selección por parte de los animales cultivados a tener en sus músculos estos AA. De la misma manera ocurrió con los AA ramificados. Mientras que los AA aromáticos no están enriquecidos en los camarones cultivados como tampoco ocurre con los aminados. El mayor contenido de los AAs Asp+Glu muestra la necesidad de generar estos AA como sustrato que permita sostener el estrés que genera el cultivo, y en el ambiente cuando estos se encuentran en situaciones extremas como pueden ser inundaciones, sequías extremas, limitaciónes tróficas, elementos xenobióticos (Carvahlo et al., 2016). Los AA indispensables enriquecidos fueron principalmente Val, Ile, y Lys, mientras que entre los aminoacidos no indispensables, los ácidos aspárticos y glutámicos fueron los que aumentaron sus porcentajes junto con Cys y Gly. Esta especie pudo incrementar porcentualmente en el músculo algunos aminoacidos que estaban en menor concentración en las dietas suministradas. Principalmente, estos fueron aquellos aminoáci- dos escenciales proteinogénicos, los que están mas asociados con el crecimiento como asi también aquellos que se relaciona con la neurotransmisión y actividad antioxidante (Elmadfa \& Meyer, 2017). Por otra parte, el perfil de AA en los ejemplares obtenidos del ambiente muestra una buena calidad nutricional de este recurso para la alimentación humana (Mildward, 2012).

Desde el punto de vista de la acuicultura, y reconociendo que las especies omnívoras son difíciles de emular en todas sus características intrínsecas durante todo el ciclo biológico, el presente trabajo pudo reconocer que, aunque el alimento suministrado en un cultivo no presente el perfil de AA de la especie, este camarón enriquece algunos de ellos cubriendo completamente las necesidades de la especie observado a partir de las altas supervivencias durante la experiencia. La manipulación que se puede realizar en la alimentación de los cultivos permite que ante la deficiencia en alguna dieta sumnistrada y que pueda ocurrir en un cultivo, la especie supla y genere caracteristicas que permita mantener la calidad de ese producto y que sea un elemento nutricional diferenciado de alto valor.

Los crustáceos, y en especial los camarones, tienen una reconocida aptitud de sus partes comestibles que permiten considerarlos como alimentos de alta calidad, tanto por sus características intrínsecas (macromoléculas de alto valor biológico) como también por su costo. Este grupo es indicado como un producto comestible de alto valor nutricional, junto al factor económico o la expectativa de ganancia monetaria potencial que podría generar su producción y comercialización. La calidad del producto es indicada en relación con la alta concentración de proteína que presenta la parte comestible del camarón, junto con la baja concentración de lípidos. Esta combinación es apreciada y puede considerarse como un recurso que puede brindar un potencial servicio a la sociedad mejorando nutricionalmente a la población, a raíz de tener en cuenta el perfil de los aminoácidos presentes. A esta característica se le suma que este camarón habita la cuenca del río Paraná y está presente en densidades que en ciertos momentos supera los $500 \mathrm{ind} / \mathrm{m}^{2}$ (Collins et al., 2007) en una superficie mayor a $2.610^{6} \mathrm{~km}^{2}$ que correspondería al menos al valle de inundación 
del río Paraná en su tramo medio (Iriondo \& Paira, 2007), aunque ese valor podría ser mucho más alto de acuerdo a los límites que se haga referencia y que corresponde a la distribución de esta especie. En toda esta superficie puede potencialmente ser utilizado este camarón en acuicultura además de realizar actividades extractivas.

Si además del volumen potencial de cultivo y de extracción, le sumamos el patrón de aminoácidos aportado por este camarón y que cubre las necesidades requeridas por el hombre en su edad niño-adolescente y adulta (WHO/FAO/UNU, 2007) debemos reconocer un recurso aún desestimado en la región. La riqueza del recurso para la alimentación humana surge al compararse con el requerimiento proteico promedio de $0.80 \mathrm{o} 0.66 \mathrm{~g}$ $\mathrm{kg}^{-1} \mathrm{~d}^{-1}$, respectivamente para cada grupo etario. La porción comestible de los camarones cultivados cubre los requerimientos de AA indispensables para el hombre, por ende, el cultivo de esta especie con dietas artificiales constituye una herramienta potencial para ser utilizada en la nutrición humana, como otro recurso que se suma al servicio ecosistémico que brinda el río Paraná.

La población en ciertos lugares del mundo manifiesta desconocimiento de los alimentos potenciales que tienen a su alcance. Esto ocurre en muchas ocasiones cuando históricamente el alimento utilizado por la población ha sido de otro tipo y no corresponde a las comidas tradicionales o de la región. En Argentina sucede esta situación, en donde la población es una consumidora masiva de carne vacuna y en menor medida porcina y avícola, dejando a solo consumos esporádicos a los provenientes de los ambientes acuáticos. Sin embargo, los alimentos de origen acuático tienen un gran valor nutricional y son utilizados masivamente en otras partes del mundo o etnias. Por ello, es necesario reconocer que muchos de estos alimentos están siendo redescubiertos por los pobladores locales, no solo para su consumo sino también para su venta como un producto que genere riqueza a la población (Godfray et al., 2010).

Para lograr que estos sean identificados, y que no tengan riesgos de que las poblaciones naturales sean sobreexplotadas es necesario realizar trabajos y evaluaciones que permitan reconocer la capacidad que tienen los ambientes de soportar la presión de extracción de esas especies para su utilización (Magalhaes et al., 2016) o la posibilidad de cultivo masivo. Cada especie en el ambiente natural tiene una dinámica que está definida por diferentes factores, abióticos y bióticos, siendo la temperatura y el ciclo hidrosedimentológico los principales factores en estos ríos de llanura. Esto determina variaciones en las tallas y tamaños poblacionales en los diferentes momentos del año (Collins et al., 2007), que permitirían cierta dinámica en la captura de ejemplares en el ambiente natural.

El valor nutricional de los recursos naturales debe ser valorado y más aún si requerimos que ese servicio tenga determinada calidad diferenciada, seguridad y previsibilidad (Butler \& Kosura, 2006). Los camarones son un alimento con características nutricionales reconocidas por su alto contenido proteico (New, 1990); esto es un servicio ecosistémico (MEA, 2005). Algunos de los servicios son fácilmente reconocidos por la población y, por ende, por la comunidad científica y los agentes de gestión política de cada estado. Mientras que otros, como la producción o aportes de alimentos, son ignorados o subvalorados, aunque sean de importancia para el bienestar, la salud, el sustento y la supervivencia de los pueblos (Costanza et al., 1997; MEA, 2005).

Algunos servicios pueden estar relacionados entre sí, por lo que podría existir sinergias entre ellos, debiendo atenderse en la valoración integral para que este sea un manejo sostenible y sustentable. Este razonamiento es de gran importancia y permite la correcta ponderación para alcanzar el cuidado del ambiente y de los servicios que éste puede brindar (Sandifer et al., 2015). Por ejemplo, la producción y/o recolección de alimento (ej. extracción y/o cultivo) es considerada en un contexto general, pero no se reconoce aún su calidad. Esta es pobremente tenida en cuenta, y más aún en los ambientes acuáticos continentales (De Groot et al., 2012; Pandeya et al., 2016).

\section{AGRADECIMIENTOS}

El presente trabajo fue realizado gracias al financiamiento obtenido del proyecto PICT2014-1996 Collins. 


\section{BIBLIOGRAFÍA}

ALAIZ, M., J. NAVARRO, J. GIRON \& E. VIOQUE. 1992. Amino acid analysis by high performance liquid chromatography after derivatization with diethylethoxymethylene-malonate. Journal of Chromatography, 591:181-186. DOI: 10.1016/0021-9673(92) 80236-N

BLANCHETTE, M. L., A. M. DAVIS, T. D. JARDINE \& R. G. PEARSON. 2014. Omnivory and opportunism characterize food webs in a large dry-tropics river system. Freshwater Science, 33(1):142-158. DOI: 10.1086/ 674632

BUTLER, C. D. \& W. O. KOSURA. 2006. Linking Future Ecosystem Services and Future Human Well-being. Ecology and Society, 11(1): 30 [online] URL: http://www. ecologyandsociety.org/vol1 1/iss 1/art30/

CARVALHO, D., V. WILLINER, F. GIRI, C. VACCARI \& P. A. COLLINS. 2016. Quantitative food webs and invertebrate assemblages of a large River: a spatiotemporal approach in floodplain shallow lakes. Marine and Freshwater Research, 68(2):293-307. DOI: 10.1071/MF15095

COLLINS, P. A. 1999. Role of natural productivity and artificial feed in enclosures with the freshwater prawn, Macrobrachium borellii (Nobili, 1896). Journal of Aquaculture in the Tropics, 14(1):47-56.

COLLINS, P. en prensa. Trophic ecology. In: Aeglidae: life history and conservation status of unique freshwater anomuran decapods. S. Santos S \& S. Bueno S. (Eds.) CRC/Taylor \& Francis Group Publishing.

COLLINS, P. A. \& J. C. PAGGI. 1998. Feeding ecology of Macrobrachium borellii (Nobili) (Decapoda: Palaemonidae) in flood valley of river Parana Argentina. Hydrobiologia, 362:21-30. DOI:10.1023/A:1003166116086

COLLINS, P., V. WILLINER \& F. GIRI. 2006. Trophic relationships in Crustacea Decapoda of a river with floodplain. In: Predation in Organisms: A Distinct phenomenon A.M.T. Elewa (Ed.):59-86. Springer Verlag, Heidelberg.

COLLINS, P., V. WILLINER \& F. GIRI. 2007.
Littoral Communities. Macrocrustaceans. In: The Middle Paraná River: Limnology of a subtropical wetland. M.H. Iriondo, J.C. Paggi \& M.J. Parma (Eds.): 277-301. Springer Verlag, Heidelberg.

COSTANZA, R., R. D'ARGE, R.D. GROOT, S. FARBERK, M. GRASSO, B. HANNON, K. LIMBURG, S. NAEEM, R.V. O'NEILL, J. PARUELO, R.G. RASKIN, P. SUTTON \& M. BELT. 1997. The value of the world's ecosystem services and natural capital. Nature, 387: 253-260. DOI: 10.1038/387253a0

DE GROOT, R., L. BRANDER, S. VAN DER PLOEG, R. COSTANZA, F. BERNARD, L. BRAAT, M. CHRISTIE, N. CROSSMAN, A. GHERMANDI, L. HEIN, S. HUSSAIN, P. KUMAR, A. MCVITTIE, R. PORTELA, L.C. RODRIGUEZ, P. TEN BRINK \& P. VAN BEUKERING. 2012. Global estimates of the value of ecosystems and their services in monetary units. Ecosystem Services, 1:50-61. DOI: 10.1016/j.ecoser.2012.07.005

ELMADFA, I. \& A. MEYER. 2017. Animal Proteins as Important Contributors to a Healthy Human Diet. Annual Review of Animal Bioscience, 5:111-131. DOI: 10.1146/annurevanimal-022516-022943

FAO. 1997. Aquaculture development. FAO Technical Guidelines for Responsible Fisheries. No. 5. FAO, Rome, Italia.

FROST, P. C., W. F. CROSS \& J. P. BENSTEAD. 2005. Ecological stoichiometry in freshwater benthic ecosystems: an introduction. Freshwater Biology, 50:1781-1785. DOI: $10.1111 / \mathrm{j} .1365-2427.2005$. 01457.x

GARCÍA-GUERRERO, M., I. S. RACOTTA \& H. VILLARREAL. 2010. Variation in lipid, protein, and carbohydrate content during the embryonic development of the crayfish Cherax quadricarinatus (Decapoda: Parastacidae). Journal of Crustacean Biology, 23(1):1-6. DOI: $10.1163 / 20021975-99990308$

GODFRAY, H. C. J., J. R. BEDDINGTON, I. R. CRUTE, L. HADDAD, D. LAWRENCE, J. F. MUIR, J. PRETTY, S. ROBINSON, S. M. THOMAS \& C. TOULMIN. 2010. Food Security: The Challenge of Feeding 9 Billion People. Science, 327:812; DOI: 10.1126/science. 1185383 
IRIONDO, M., J. C. PAGGI \& J. PARMA. 2007. The Middle Paraná River: Limnology of a subtropical wetlands. Springer Berlin, New York.

IRIONDO, M. H. \& A. PAIRA. 2007. Physical Geography of the Basin. In: The Middle Paraná River: Limnology of a subtropical wetland. M.H. Iriondo, J.C. Paggi \& M.J. Parma (Eds.): 7-32. Springer Verlag, Heidelberg.

ISLAM, A., S. MONDAL, S. BHOWMIK, S. ISLAM \& M. BEGUM. 2017. A comparative analysis of the proximate composition of wild and cultured prawn (Macrobrachium rosenbergii) and shrimp (Penaeus monodon). International Journal of Fisheries and Aquatic Studies, 5(4):59-62.

JUNK, W. J., P. B. BAYLEY \& R. E. SPARKS. 1989. The flood pulse concept in river-floodplains systems. Canadian Special Publication Fisheries Aquatic Science, 106:110-127.

KRIVAN, V.\& S. DIEHL. 2005. Adaptive omnivory and species coexistence in tri-trophic food webs. Theoretical Population Biology, 67:85-99. DOI: 10.1016/j.tpb.2004.09.003

LAUGHLIN, R. A. 1982. Feeding habits of the crab, Callinectes sapidus Rathbun, in the Apalachiola estuary, Florida. Bulletin of Marine Science, 32:807-822.

MAGALHAES, C., M. ROCHA CAMPOS, P. COLLINS \& F. MANTELATTO. 2016. Diversity, distribution and conservation of freshwater crabs and shrimps in South America. In: A global overview of the conservation of freshwater decapod crustaceans. T. Kawai \& N. Cumberlidge (Eds.): 303-322. Springer Verlag, Heidelberg.

MEA, 2005. Millenium Ecosystems Assesment. Ecosystems and Human Well-Being: Synthesis. Island Press, Washington, DC.

MILLWARD, D. J. 2012. Amino acid scoring patterns for protein quality assessment. British Journal of Nutrition, 108:31-43. DOI: 10.1017/S0007114512002462

NEW, M. B. 1990. Freshwater prawn culture: a review. Aquaculture, 88: 99-143. DOI: 10.1016/0044-8486(90)90288-X

PANDEYA, B., W. BUYTAERTA, Z. ZULKAFLIB, T. KARPOUZOGLOUD, F. MAOE \&
D. M. HANNAHE. 2016. A comparative analysis of ecosystem services valuation approaches for application at the local scale and in data scarce regions. Ecosystem Services, 22:250-259. DOI: 10.1016/j.ecoser. 2016.10.015

RAMÍREZ, E., A. SILVA, M. GUEVARA, M. NÚÑEZ, R. BAUZA \& B. ARREDONDO-VEGA. 2010. Composición bioquímica del camarón dulceacuícola Macrobrachium jelskii (Miers, 1877) sometido a condiciones de cultivo. Zootecnia Tropical, 28(1):65-72.

RANGAPPA, A., T. RAJ KUMAR, P. JAGANMOHAN \& M. SRINIVASULU REDDY. 2012. Studies on the Proximal Composition of Freshwater Prawns Macrobrachium rosenbergii and Macrobrachium malcomsonii. World Journal of Fish and Marine Sciences, 4 (2): 218-222. DOI: 10.5829/idosi.wjfms.2012. 04.02.61223

SABOROWSKI, R. 2015. Nutrition and digestion. In: Physiology, vol. 4. The Natural History of the Crustacea. E.S. Chang \& M. Thiel (Eds.): 285-319. Oxford University Press.

SANDIFER, P. A., A. E. SUTTON-GRIER \& B. P. WARD. 2015. Exploring connections among nature, biodiversity, ecosystem services, and human health and well-being: Opportunities to enhance health and biodiversity conservation. Ecosystem Services,12:1-15. DOI: 10.1016/j.ecoser.2014.12.007

TACON, A. G. 2016. Nutrición y alimentación de peces y camarones cultivados manual de capacitación. Programa cooperativo gubernamental FAO, Italia gcp/rla/102/ita proyecto Aquila ii, Documento de campo no 4.

WHO/FAO/UNU (World Health Organization / Food and Agriculture Organization / United Nations University). 2007. Protein and Amino Acid Requirements in Human Nutrition Report of a Joint WHO/FAO/UNU Expert Consultation. WHO Technical Report Series no. 935. Geneva: WHO.

WINEMILLER, K. O. 2004. Floodplain river food webs, generalizations and implications for fisheries management. In: Proceedings of the Second International Symposium on the Management of Large Rivers for Fisheries Volume 2. R.L. Welcomme \& T. Petr (Eds.): 
285-309. Phnom Penh, Cambodia. RAP Publication.
ZAR, J. H. 1996. Biostatistical Analysis. Prentice Hall: New York.

Con el patrocinio de:

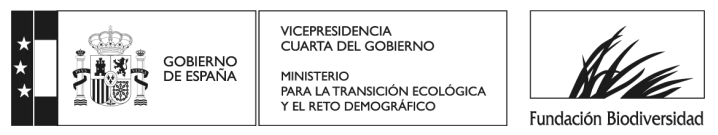

